\title{
UMAP 2018 Fairness in User Modeling, Adaptation and Personalization (FairUMAP 2018) Chairs' Welcome
}

It is our great pleasure to welcome you to the UMAP 2018 FairUMAP workshop. This full-day workshop brings together researchers working at the intersection of user modeling, adaptation, and personalization on one hand, and bias and fairness in machine learning on the other hand.

The workshop was motivated by the observation that these two fields increasingly impact on each other: Personalization has become a ubiquitous and essential part of systems that help users find relevant information in today's highly complex information-rich online environments. Machine learning, recommender systems, and user modeling are key enabling technologies that allow intelligent systems to learn from users and adapt their output to users' needs and preferences. However, there has been a growing recognition that these underlying technologies raise novel ethical, legal, and policy challenges. It has become apparent that a single-minded focus on the user preferences has obscured other important and beneficial outcomes such systems must be able to deliver. System properties such as fairness, transparency, balance, openness to diversity, and other social welfare considerations are not captured by typical metrics based on which data-driven personalized models are optimized. Indeed, widely-used personalization systems in such popular sites such as Facebook, Google News and YouTube have been heavily criticized for personalizing information delivery too heavily at the cost of these other objectives.

Bias and fairness in machine learning are topics of considerable recent research interest. However, more work is needed to expand and extend this work into algorithmic and modeling approaches where personalization is of primary importance. The goal of this workshop was to bring together a growing community of experts from academia and industry to discuss ethical, social, and legal concerns related to personalization, and specifically to explore a variety of mechanisms and modeling approaches that help mitigate bias and achieve fairness in personalized systems.

We invited papers on a range of topics including Bias and discrimination in user modeling, personalization and recommendation; Computational techniques and algorithms for fairnessaware personalization; Definitions, metrics and criteria for optimizing and evaluating fairnessrelated aspects of personalized systems; Data preprocessing and transformation methods to address bias in training data; User modeling approaches that take fairness and bias into account; User studies to evaluate impact of personalization on fairness, balance, diversity, and other social welfare criteria; Balancing needs of multiple stakeholders in recommender systems and other personalized systems; and 'Filter bubble' or 'balkanization' effects of personalization; Transparent and accurate explanations for recommendations and other personalization outcomes.

These topics were addressed by six contributed papers and two invited talks by Judy Kay from University of Sydney and Robin Burke from DePaul University. More details about these talks can be found on the workshop Website: https://fairumap.wordpress.com/.

Two contributed papers focus directly on questions of how to generate "fair" recommendation sets.

Karako and Manggala address the trade-off between relevance and fairness in personalized recommendations, where "fair" means a balanced representation across demographics in 
recommended items. They present a fairness-aware variation of the Maximal Marginal Relevance re-ranking method which uses representations of curated labeled image dataset of demographic groups computed using a pre-trained deep convolutional neural network. Their results indicate that the method is robust against the amount of curated labeled data used to compute the representations, and is therefore suited as a post-processing step for recommender systems and search.

Zheng, Dave, Mishra and Kumar study fairness in recommendation, where "fair" means that the expectations and preferences not only of the users but also of other stakeholders are integrated. They perform exploratory analysis on speed-dating data, where the user expectations are clearly defined. They build a multi-dimensional utility framework by utilizing multi-criteria ratings, and demonstrate that they are able to obtain a successful tradeoff between utility optimizations and recommendation performance.

Four contributed papers focus on how to support diversity in media, where an even larger set of stakeholders contributes to what is recommended and which of these recommendations is "used".

Hirschmeier and Beule focus on the producer side. They ask how media institutions with a legal mandate to present a diversified programme can act when media use is increasingly personalized. They propose an approach for selectively avoiding filter bubbles in personalized radio consumption, and develop a framework that helps to assess the compliance of personalized radio offers with public mandates.

Peperkamp and Berendt also focus on the producer side. Their goal is to improve diversity in news reporting by making recommendations to journalists. They propose a way of measuring diversity by focusing on actor diversity in an ontology-based text mining approach, and present an initial version of their Diversity Checker tool and first evaluation results.

Tintarev, Sullivan, Guldin, Qiu, Odjik, Permadi and Pangaribuan focus on the news consumer side. Their approach aims at automatically identifying content that represents a wider range of opinions on a given topic. The method was evaluated in an offline way and with users. Results suggest that the hand-crafted distance function, using linguistic and stylometric terms, influences diversity in terms of topic and channel, but that news readers may not necessarily have an intrinsic interest in diversified news.

Hirschmeier et al.'s, Peperkamp et al.'s and Tintarev et al.'s results indicate that filter bubbles need to be studied and addressed along the whole chain of news production and consumption, and that the recommender approach itself is a major challenge in these various settings. "Breaking" filter bubbles remains an algorithmic and interface-design challenge.

Babaei, Chakraborty, Kulshrestha, Redmiles, Cha and Gummadi focus on "diversities" associated with fake news. They analyze biases in the perception of truth in news stories and their implications for fact checking. They used online user surveys to measure how readers perceive truth in viral news stories. Their findings suggest that the stories most in need of being factchecked are the stories where users exhibit the largest perception biases, which may require a rethinking of current fact-checking focuses on stories based on their actual truth level.

More details about the workshop, including the workshop organization, accepted papers and invited talks are available at the workshop Web site: https://fairumap.wordpress.com/.

$\begin{array}{llll}\text { Bamshad Mobasher } & \text { Robin Burke } & \text { Michael Ekstrand } & \text { Bettina Berendt } \\ \text { Co-Chair } & \text { Co-Chair } & \text { Co-Chair } & \text { Co-Chair } \\ \text { DePaul University } & \text { DePaul University } & \text { Boise State University } & \text { KU Leuven }\end{array}$




\title{
FairUMAP 2018 Organization and Program
}

\author{
Workshop Bamshad Mobasher (DePaul University, USA) \\ Chairs: $\quad$ Robin Burke (DePaul University, USA) \\ Michael Ekstrand (Boise State University, USA) \\ Bettina Berendt (KU Leuven, Belgium) \\ Program Solon Barocas (Cornell University, USA) \\ Committee: Pablo Castells (Universidad Autónoma de Madrid, Spain) \\ David Chin (University of Hawaii, USA) \\ Jonathan Gemmell (DePaul University, USA) \\ Krishna Gummadi (MPI-SWS, Germany) \\ Sara Hajian (Eurecat, Technology Center of Catalonia, Spain) \\ Eelco Herder (Radboud Univeristy, The Netherlands) \\ Dietmar Jannach (AAU Klagenfurt, Austria) \\ Toshihiro Kamishima (AIST, Japan) \\ Daniel Kluver (University of Minnesota / Macalester College, USA) \\ Bart Knijnenburg (Clemson University, USA) \\ Suresh Venkatasubramanian (University of Utah, USA) \\ Yong Zheng (Illinois Institute of Technology, USA) \\ Yair Zick (National University of Singapore, Singapore)
}

Accepted

- $\quad$ Nava Tintarev, Emily Sullivan, Dror Guldin, Sihang Qiu, Daan Odjik, Reza Aditya

Papers: Permadi and Andreas Christian Pangaribuan. Same, Same, but Different:

Algorithmic Diversification of Viewpoints in News

- Stefan Hirschmeier and Vanessa Beule. Compliance of Personalized Radio with Public Mandates

- Mahmoudreza Babaei, Abhijnan Chakraborty, Juhi Kulshrestha, Elissa M. Redmiles, Meeyoung Cha and Krishna Gummadi. Analysing. Biases in Perception of Truth in News Stories and their Implications for Fact Checking

- Chen Karako and Putra Manggala. Using image fairness representations in diversity-based re-ranking for recommendations

- Yong Zheng, Tanaya Dave, Neha Mishra and Harshit Kumar. Fairness In Reciprocal Recommendations: A Speed-Dating Study

- Jeroen Peperkamp and Bettina Berendt. Diversity Checker: toward recommendations for improving journalism with respect to diversity

Invited - Judy Kay. Deep and holistic fairness, accountability and user control in Talks: $\quad$ user models and personalisation

- Robin Burke. What's hard about fair recommendation? 